\title{
Investigation of shock attenuation in a shock tube using DSMC method
}

Cite as: AIP Conference Proceedings 2132, 070012 (2019); https://doi.org/10.1063/1.5119566

Published Online: 05 August 2019

Xiaowei Xue, Song Chen, and Quanhua Sun

\section{ARTICLES YOU MAY BE INTERESTED IN}

DSMC simulations of turbulent flows at moderate Reynolds numbers

AIP Conference Proceedings 2132, 070010 (2019); https://doi.org/10.1063/1.5119564

DSMC simulation of gas heat transfer between coaxial cylinders

AIP Conference Proceedings 2132, 070011 (2019); https://doi.org/10.1063/1.5119565

Non-equilibrium parameter for a hybrid Fokker-Planck/DSMC scheme

AIP Conference Proceedings 2132, 070002 (2019); https://doi.org/10.1063/1.5119556

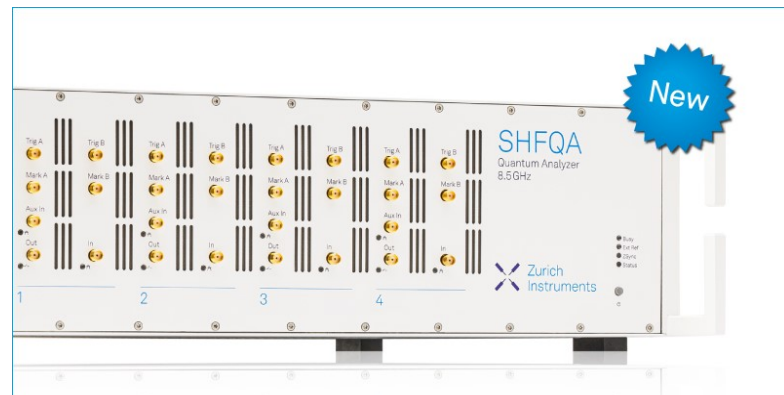

\section{Reynolds numbers
https://doi.org/10.1063/1.511956
https://doi.org/10.1063/1.5119565




\title{
Investigation of Shock Attenuation in a Shock Tube Using DSMC Method
}

\author{
Xiaowei Xue ${ }^{1,2, \text { a) }}$, Song Chen $^{3, b)}$ and Quanhua $\operatorname{Sun}^{1,2, c)}$ \\ ${ }^{1}$ State Key Laboratory of High Temperature Gas Dynamics, Institute of Mechanics, CAS, Beijing 100190, China \\ ${ }^{2}$ School of Engineering Science, University of Chinese Academy of Sciences, Beijing 100049, China \\ ${ }^{3}$ Department of Mechanics and Aerospace Engineering, Southern University of Science and Technology, Shenzhen \\ 518055, China \\ a)xuexiaowei@imech.ac.cn \\ ${ }^{b)}$ chens@sustc.edu.cn \\ c)qsun@imech.ac.cn
}

\begin{abstract}
Shock waves propagating in shock tubes or small channels will be constantly attenuated during propagation due to viscous effect and wall heat conduction. The physical mechanism of this attenuation process has not been clearly discussed in the literature although many investigations have studied the laws and extent of shock wave attenuation under various initial conditions. In this work, the DSMC method is employed to study the propagation and attenuation of shock waves in a 2D planar tube. Numerical results show that the thickness of the unsteady velocity boundary layer in the shock coordinate increases nearly proportional to the square root of time whereas the disturbance of wall effects propagates approximately with the sound velocity behind the shock wave. Appreciable shock curvature is clearly seen in the shock wave and boundary layer interaction corner, which is found to be oscillated. Further analysis shows that the oscillation characteristic of the shock profile is caused by the periodic propagation of low-pressure disturbance generated from the wall surface. Finally, shock wave is found to be more severely attenuated in narrower tube or having larger Mach number at the same time moments.
\end{abstract}

\section{INTRODUCTION}

Due to viscous effect and wall heat conduction, the flow properties in the actual shock tube will deviate from the ideal shock tube theory. An unsteady boundary layer will be induced when the shock wave and expansion waves propagate to both ends of the tube. As time goes by, the contact surface speeds up while shock wave decelerates gradually. In particular, this attenuation behavior will be greatly enhanced for smaller scale or low-pressure shock tubes. For shock tube experiments, shock attenuation will significantly reduce the effective experimental time, and may further lead to shock bifurcation and "driver gas contamination". Therefore, to better understand the shock tube flow, it is necessary to study the physical mechanism of shock propagation and attenuation.

In the past, many studies, including experiments, theoretical modeling and numerical simulations, have been done on this phenomenon. Early experimental results found, when the driven section pressure is sufficiently low with a fixed shock tube diameter, the shock velocity will decrease significantly over time, and the actual effective experimental time will no longer grow linearly with the length of the shock tube [1-3]. Later, researchers like Sun [4] and Brouillette [5] have conducted different experiments to investigate the scale effect in microscale shock tubes or channels.

In 1960s, Duff [1] and others [2,3,6] started the modeling work on shock tube performance at low initial pressure. Recently, Brouillette [5] developed a 1D control volume model that included explicitly the effects of viscosity and heat transfer in source terms. A dimensionless scaling factor $S c$ was proposed to analyze the scale effect. However, as Duff [1] pointed out earlier, it is not enough to simplify the diffusion phenomena as small disturbances in the 1D model; 2D unsteady flow analysis should be considered in order to address typical characteristics of this viscousoriented flow. 
As for numerical simulations, both continuum-based and particle-based approaches have been employed to study the shock attenuation problem. Zeitoun [7-9] used several techniques, including the N-S equation method with or without the velocity slip and temperature jump boundary conditions, DSMC method and BGK model equation method, to study the shock attenuation under different Knudsen numbers and initial shock strength. Two power-law fitting formulas of shock Mach number attenuation as function of an improved local scaling factor $S c_{x}$ were given for laminar and turbulent flows respectively. Watvisave and Puranik [10] investigated wall effects in a microscale shock tube using a hybrid MD-DSMC algorithm. It was noticed that the extent of wall accommodation significantly affects the attenuation behavior of shock waves, and the diffusive reflection model seems to overestimate the viscous effect. Deshpand and Puranik [11] later studied the effects of viscosity and wall heat conduction on shock attenuation in narrow channels. Their results indicated that the thermal conductivity and thickness of the wall material have negligible effects on the shock attenuation during the time of interest.

In general, previous studies are mainly focused on the behavior of shock wave attenuation under different initial conditions, while the physical mechanism of wall effects on this shock attenuation process and detail description of unsteady flow structures have not been well understood yet. In this study, the DSMC method is employed to study the kinetic behavior of 2D planar shock wave attenuation in shock tubes.

\section{PROBLEM SETUP}

The standard DSMC method is employed to study the propagation and attenuation of a shock wave in a 2D planar tube. Initial conditions of simulation cases are listed in Table 1 , where $H_{0.5}$ refers to half height of the tube simulated. Figure 1 shows the sketch of the simulation. Each simulation is performed in an initial shock coordinate system and only the lower half of the tube is simulated due to geometric symmetry. The left and right boundary conditions are set as outlet and inlet, respectively. The upper boundary is the symmetry line, while the lower boundary is set as the moving wall, with the wall velocity and temperature identical to the undisturbed flow property. Initial parameters on both sides of the shock wave are set using the Ranking-Hugoniot relations. The simulated gas is argon, thus the real gas effects are not included. The DSMC method employs the VHS molecular model for collisions and Maxwellian reflection model on the wall. The wall accommodation coefficient is initially set to 0 until the shock structure is fully developed. It is adjusted to 1 later to account the wall effects.

TABLE 1. The initial conditions of different simulation cases

\begin{tabular}{rcccccc}
\hline Case & $\mathrm{P}_{1}(\mathrm{~Pa})$ & $\mathrm{P}_{2}(\mathrm{~Pa})$ & $\mathrm{T}_{1}(\mathrm{~K})$ & $\mathrm{T}_{2}(\mathrm{~K})$ & $\mathrm{Ma}$ & $\mathrm{H}_{0.5}(\mathrm{~m})$ \\
\hline 1 & 8.23 & 39.09 & 298 & 619.28 & 2 & 0.5 \\
2 & 8.23 & 39.09 & 298 & 619.28 & 2 & 1 \\
3 & 8.23 & 255.09 & 298 & 2586.64 & 5 & 0.5 \\
\hline
\end{tabular}

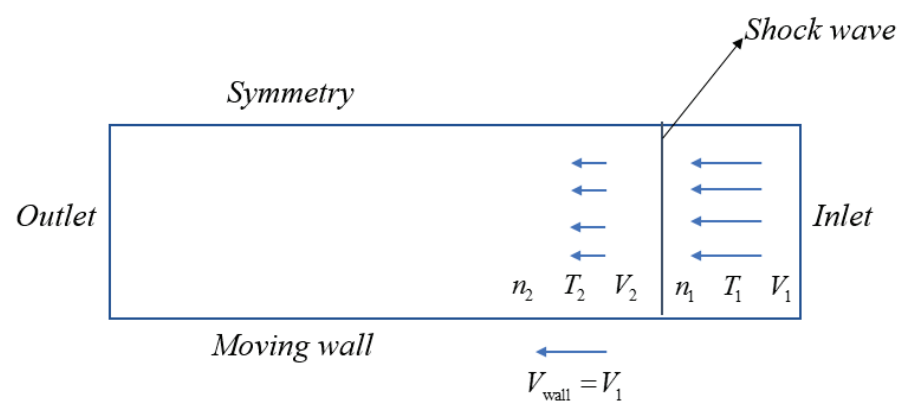

FIGURE 1. Sketch of the shock attenuation simulation.

\section{RESULTS AND DISCUSSION}

Figure 2 shows the evolution of the shock structure from initial ideal discontinuity where the normalized density contours are displayed at several time instants for the case of Mach number 2. The contours exhibit small statistical noise but random walker effect is not observed. It takes about $\mathrm{t}=50 \tau_{1}$ ( $\tau$ is the mean collision time of initial gas) to 
develop a stable shock structure as shown in Fig. 3, where the width of the shock structure is approximately 8 mean free paths of the undisturbed gas that is consistent with that in the literature [12].

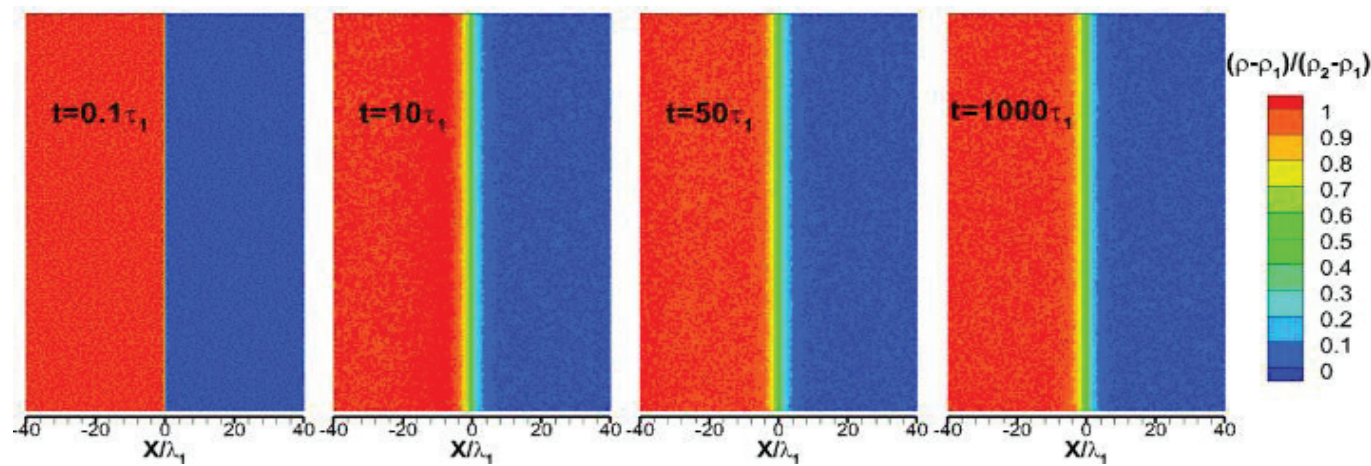

FIGURE 2. Normalized density contours showing the evolution of shock structure when Mach number is 2.

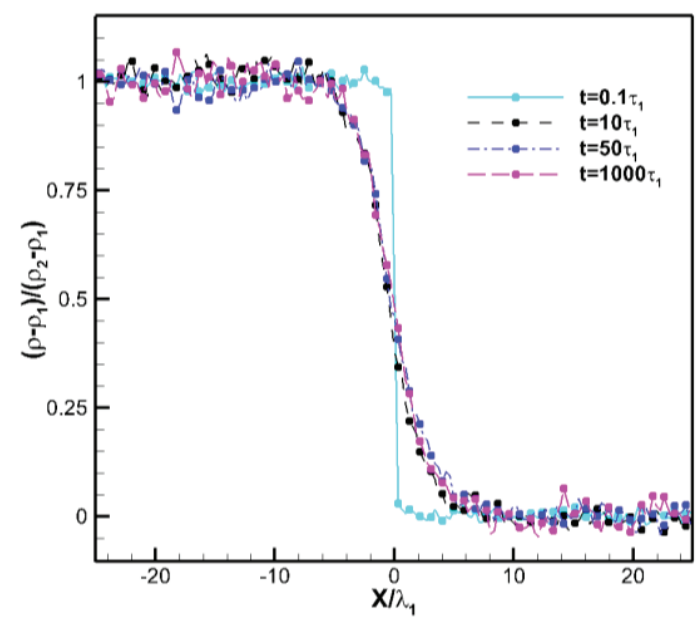

FIGURE 3. Normalized density profiles during the evolution of shock structure.

When the wall effect is applied, a boundary layer develops and interacts with the shock wave. The $\mathrm{x}$-component velocity contours for case 2 are shown in Fig. 4(a) after the wall is applied for $3100 \tau_{2}$, which indicates that the shock front is no longer straight and a boundary layer develops along the wall. The data in Fig. 4 are obtained by using time averaging over the interval of 200 time steps $\left(20 \tau_{2}\right)$. Thus the presented data are the average results over a relatively short period, which reduces the statistical noise and keeps the profile characteristics.

The thickness of the velocity boundary layer develops with the time as shown in Fig. 4(b) where the data are obtained by averaging the velocity in the domain where the boundary layer has not been affected by the shock wave (the domain far away from the shock wave). Namely, the average process here is accurate physically and reduces the statistical noise. It shows that the thickness is nearly proportional to the square root of time.

Distributions of flow properties along the direction normal to wall are shown in Fig. 5 at several time instants. The properties are normalized using the initial post-shock values except that the normal velocity is shown in its value. The data is also the average over the $\mathrm{x}$-direction in the domain where the boundary layer has not been affected by the shock wave. In general, the gas flows towards the wall and the density near the wall is increased, because the wall is relatively cool. The y-velocity induced exhibits a non-monotonic variation along the y direction due to restrictions of the upper and lower boundaries. Figure 5(a) shows the flow information at $0.1 \tau_{2}$ where the DSMC results are identical to the collisionless theory of compressible Rayleigh problem. It can be seen that significant velocity slip and temperature jump can be observed near the wall. In Figure 5(b) at $1 \tau_{2}$, the collisionless theory predicts a slightly faster disturbance than the DSMC results, which is reasonable since molecular collisions will slow the initial propagation of disturbance. All the four figures indicate the existence of a propagating low-pressure, lowtemperature disturbance zone. 


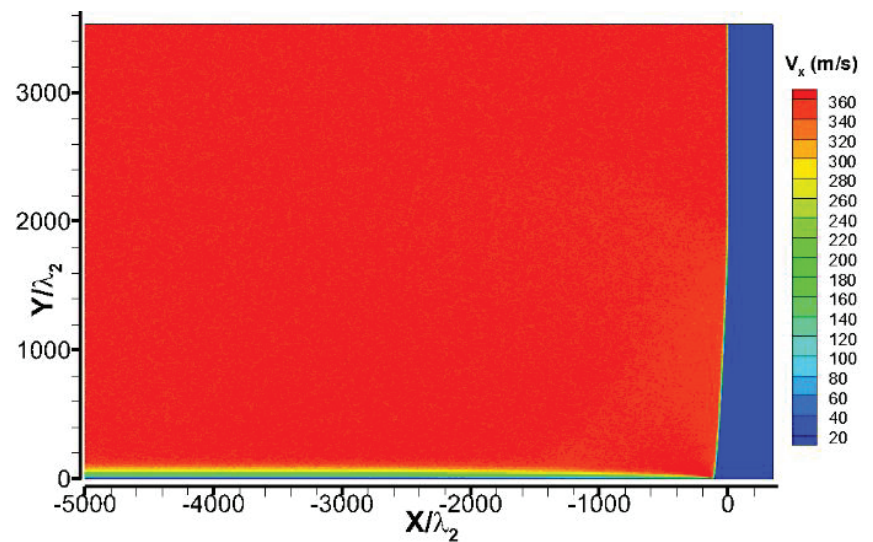

(a)

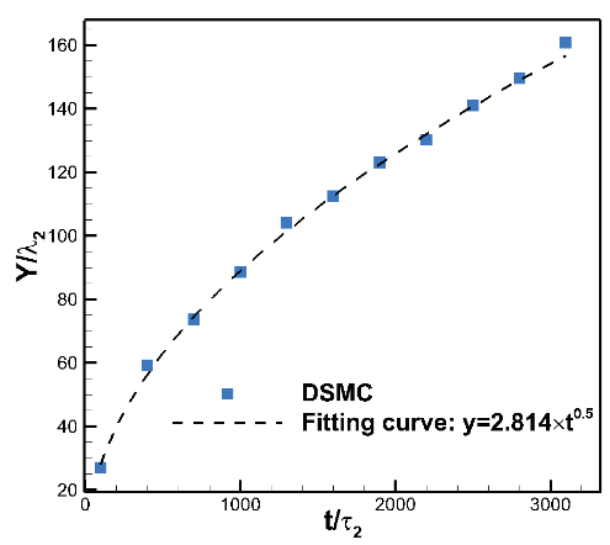

(b)

FIGURE 4. Velocity boundary layer. (a) X-component velocity contours at $t=3100 \tau_{2}$; (b) Velocity boundary layer thickness as function of time.

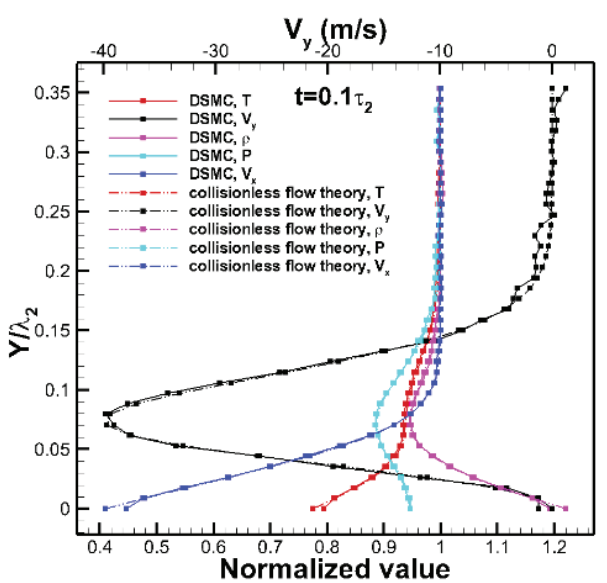

(a)

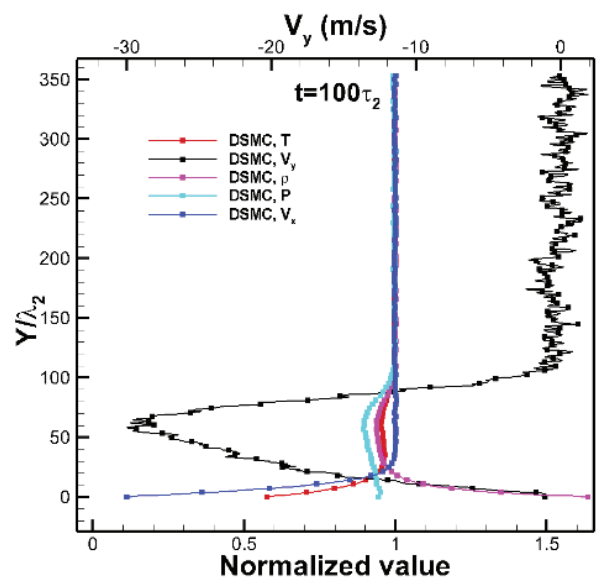

(c)

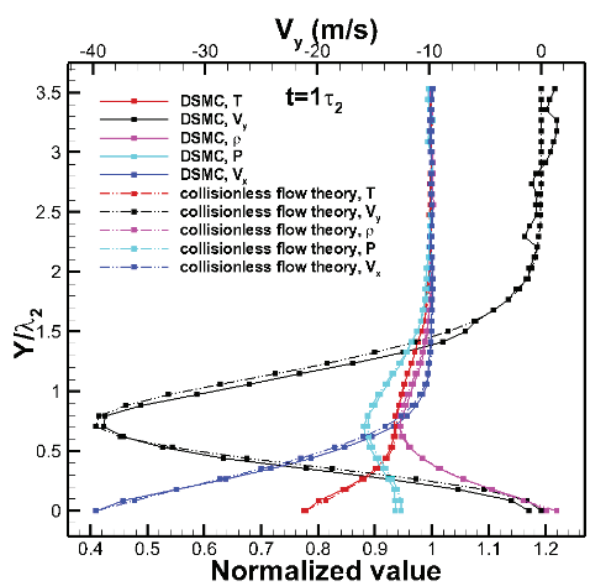

(b)

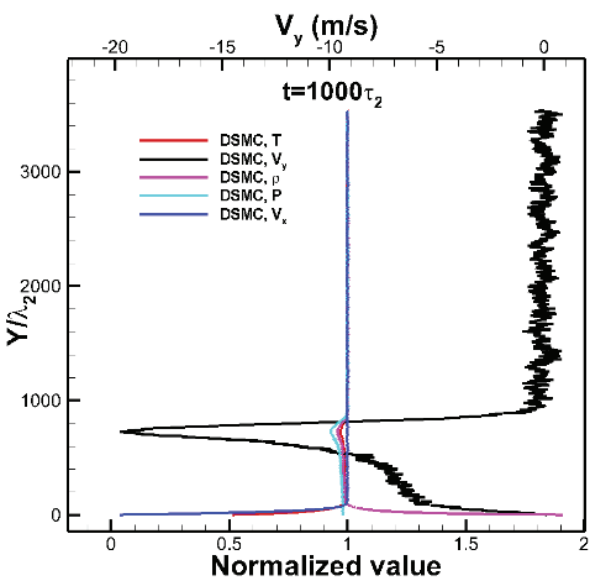

(d)

FIGURE 5. Property distributions along the y-direction at different time instants when $M a=2, H=1 \mathrm{~m}$. (a) $t=0.1 \tau_{2}$. (b) $t=1 \tau_{2}$. (c) $t=100 \tau_{2}$. (d) $t=1000 \tau_{2}$. 
The propagation of the disturbance is analyzed and is shown in Fig. 6 where the upmost position of disturbance (defined as $\mathrm{y}_{\text {perturbation}}$ ) and the maximum amplitude of the $\mathrm{y}$-component velocity are plotted over the time. It is found that the disturbance generated from wall surface propagates upward with approximately the sound velocity behind the shock, which is much faster than the development of velocity boundary layer. It can also be noted that the maximum amplitude of y-component velocity is gradually attenuated with time.

The interaction between shock wave and boundary layer is illustrated in Fig. 7 using pressure contours near the interaction corner when Mach number is 2 with $H=0.5 \mathrm{~m}$. As time elapses, appreciable shock curvature first appears near the surface and then develops upwards with time, and after the disturbance propagates to the symmetric line (center line of the shock tube), the entire shock position starts to move backwards. Namely, the shock wave gets attenuated since its position cannot keep with the initial shock position as Fig. 7 is presented in the initial shock coordinate.

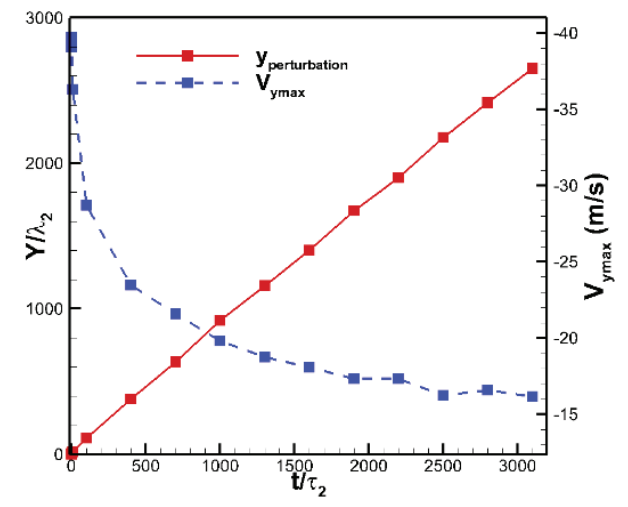

FIGURE 6. Disturbance propagation of wall effects when $M a=2, H=1 \mathrm{~m}$.

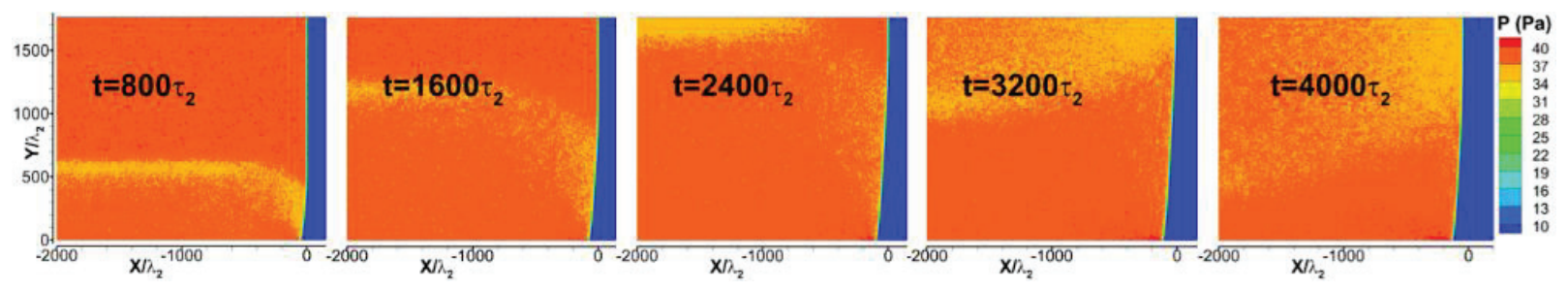

FIGURE 7. The time evolution of shock wave structure when $M a=2, H=0.5 \mathrm{~m}$.

Figure 8 shows y-component velocity and pressure contours near the shock front at $t=1600 \tau_{2}$. It is noted that large temperature discontinuity in the corner induces a large y-component velocity and molecules tend to flock to the wall, thus resulting in visible pressure decrease in bulk flow just behind the shock front. The decrease and nonuniformity of pressure cause the shock structure to bend significantly.

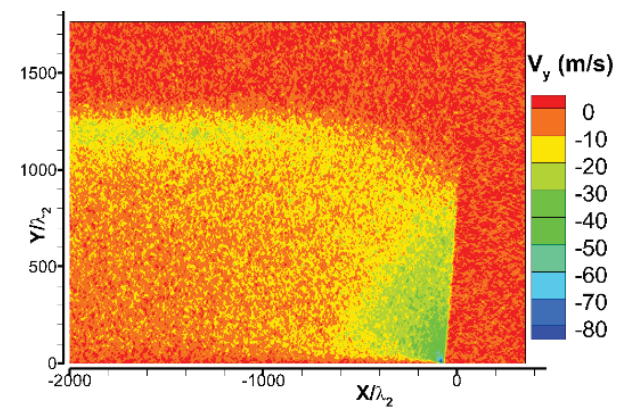

(a)

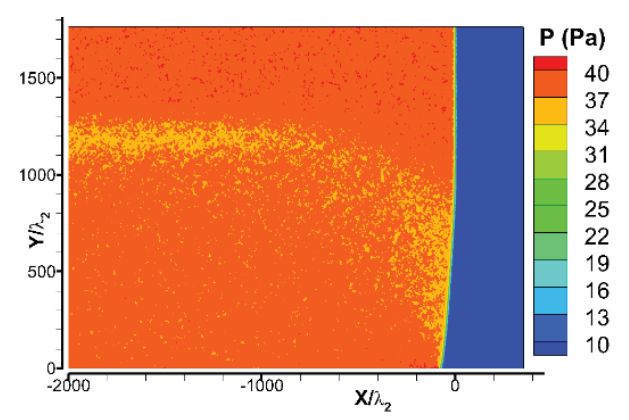

(b)

FIGURE 8. Velocity and pressure contours near the shock wave boundary layer interaction corner at $t=1600 \tau_{2}$ when $M a=2$.

(a) Velocity normal to flow. (b) Pressure. 
Figure 9 compares shock attenuation with different shock tube heights where the half height equals to $0.5 \mathrm{~m}$ and $1 \mathrm{~m}$, respectively. Evolutions of shock wave profiles with two tube heights are compared in Figure 9(a) at different time instants, with the shock wave location defined as position where the local density equals to $0.5\left(\rho_{1}+\rho_{2}\right)$ of the initial value (i.e. $1.14 \rho_{1}$ for $M a=2$ ). It can be noted that the narrower shock tube leads to more severe attenuation in shock velocity, as illustrated both in Figure 9(a) and Figure 10. The profiles of shock waves at different times exhibit an oscillation characteristic, which indicates that shock curvature is caused by the periodic propagation of low-pressure disturbances which reflect from wall surfaces. Figure 9(b) shows the evolution of normalized shock curvature factor over time (non-dimensionalized using the tube height and the initial post-shock speed of sound), which is defined as $\left(X_{\text {center }}-X_{\text {wall }}\right) / H$, where $X_{\text {center }}, X_{\text {wall }}$ are the extracted shock wave position at the symmetric line and wall surface, respectively, and $H$ is the half height of the shock tube. It is noticed that this factor exhibits obvious periodic oscillation characteristics as well and the amplitude of the oscillation is significantly attenuated over time. In the long term, it seems to gradually reach a constant value, and it is reasonable that the narrower tube has a relatively higher value of this factor. It should be noted that, as pointed out in [13], for viscous shock tube flows, the curvature of incident shock wave will induce a lateral pressure gradient and form a jet flow. Figure 10 shows the shock Mach number attenuation with different shock tube heights over time. The curves also show the nature of periodic oscillation. The smaller the scale is, the more severe the shock Mach number attenuates.

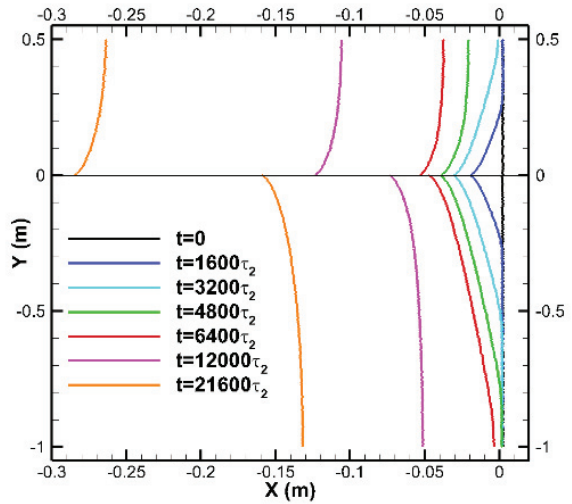

(a)

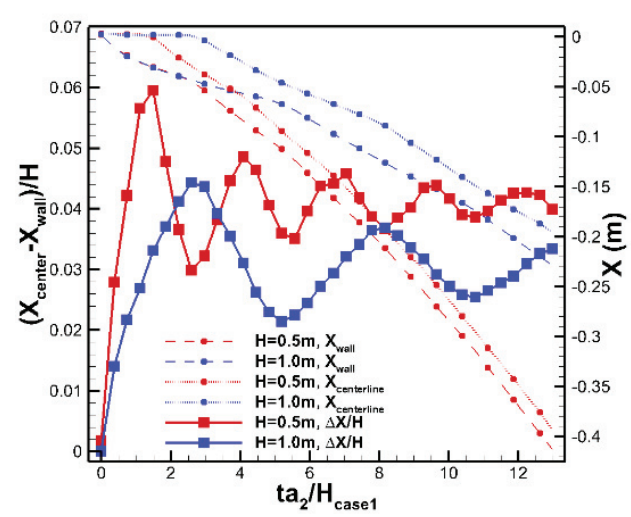

(b)

FIGURE 9. Comparison of shock attenuation with different shock tube heights. (a) Evolution of shock wave profiles at different time instants when $M a=2$. Upper: $H=0.5 \mathrm{~m}$; Down: $H=1 \mathrm{~m}$. (b) Evolution of the normalized shock curvature factor.

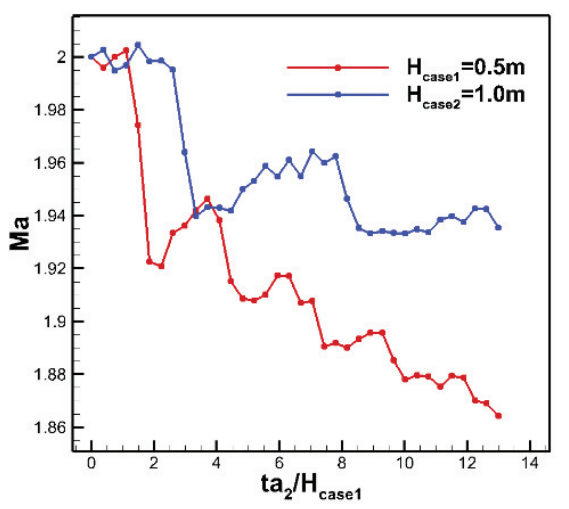

FIGURE 10. Comparison of shock Mach number attenuation with different shock tube heights.

Finally, the effects of different initial Mach number are investigated as well. Figure 11(a) shows that the normalized shock curvature factor with higher Mach number exhibits a smaller oscillation period, which is due to the effect that for the identical driven side conditions, higher Mach number corresponds to higher sound velocity behind the shock wave and leads to much faster disturbance propagation of wall effects. Interestingly, this 
normalized shock curvature factor seems to reach the identical constant value for different Mach numbers in the long run, which may indicate that the Mach number has negligible effects on this factor when compared with the scale of the tube simulated. Figure 11(b) shows that at the same time instant, higher Mach number corresponds to a more severe Mach number attenuation, which is because that shock wave travels much longer distance at the same time instant for larger initial Mach number.

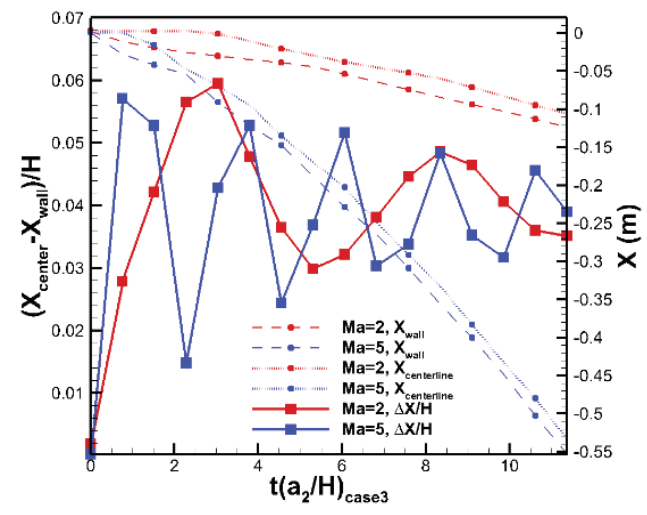

(a)

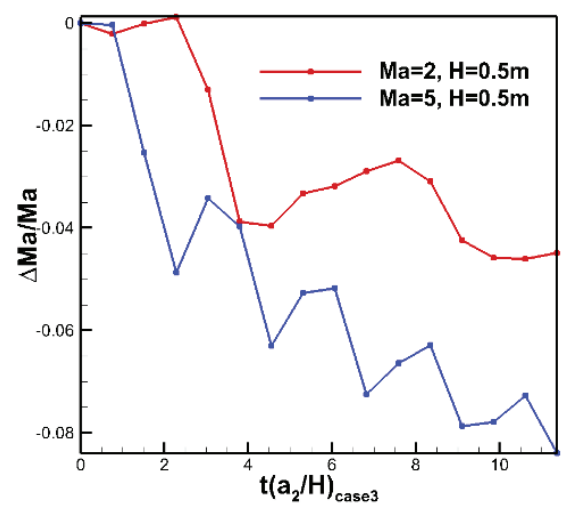

(b)

FIGURE 11. Comparison of shock attenuation with different Mach numbers. (a) Evolution of the normalized shock curvature factor. (b) Comparison of shock Mach number attenuation with different initial shock strength.

\section{CONCLUSION}

In this paper, the DSMC method is employed to simulate the propagation and attenuation of shock waves in a 2D duct or channel. Numerical results show that in the shock coordinate, the disturbance of wall effects propagates approximately with the sound velocity behind the shock wave while the thickness of the unsteady velocity boundary layer increases nearly proportional to the square root of time. Appreciable shock curvature is clearly seen in the shock front, and the oscillation characteristic of shock profile also indicates that this deformation is caused by the periodic propagation of low-pressure disturbance generated from the side wall surfaces. Finally, with identical driven conditions, shock waves will be more severely attenuated in narrower tube or with larger Mach number at the same time moments. Future study will analyze the effect of different gas-surface interaction models, shock attenuation characteristics at much higher Knudsen numbers and over a much longer period of flow time. In addition, the actual shock tube flow which includes both the effects of contact surface and expansion waves will be considered as well.

\section{ACKNOWLEDGMENTS}

This work was supported by the Strategic Priority Research Program of Chinese Academy of Sciences (Grant No.XDA17030100) and the National Natural Science Foundation of China (Grants 11372325 and 91116013).

\section{REFERENCES}

1. R. E. Duff, Phys. Fluids 2(2), 207-216 (1959).

2. A. Roshko, Phys. Fluids 3(6), 835-842(1960).

3. W. J. Hooker, Phys. Fluids 4(12), 1451-1463(1961).

4. M. Sun, T. Ogawa, and K. Takayama, Proceedings of 23rd International Symposium On Shock Waves, Fort Worth, Texas, 2001, edited by F. K. Lu (Arlington, TX, 2002), pp. 1320-1325.

5. M. Brouillette, Shock Waves 13(1), 3-12(2003).

6. H. Mirels, Phys. Fluids 6(9), 1201-1214 (1963).

7. D. E. Zeitoun, Y. Burtschell, Shock Waves 15(3-4), 241-246(2006).

8. D. E. Zeitoun, Y. Burtschell, I. A. Graur, et al. Shock Waves 19(4), 307-316(2009).

9. D. E. Zeitoun, Phys. Fluids 27(1), 011701(2015). 
10. D. S. Watvisave, B. P. Puranik, B. P. Bhandarkar, Shock Waves 26(4), 477-489(2016).

11. A. Deshpande, B. Puranik, Shock Waves 26(4), 465-475(2016).

12. G. A. Bird, Molecular Gas Dynamics and the Direct Simulation of Gas Flows (Clarendon Press, Oxford, 1994).

13. S. Chen, Ph.D. thesis, University of Chinese Academy of Sciences, Beijing, 2015. 